relevant strains of the virus to just 1 in 20 . This is a significant result. About 14 million people in the United States, most in their late teens and early 20s, become infected with HPV each year. The two most dangerous strains - those targeted by vaccines made by both Merck and GlaxoSmithKline - cause cervical cancer, other anogenital cancers and throat cancer. Merck's vaccine also protects against two further strains that cause genital warts.

The vaccine is given in three doses over six months. The CDC first recommended that all girls aged 11 and 12 be vaccinated. In 2011, it said the same for boys, for whom only the Merck vaccine is licensed. The idea is to immunize children before they become sexually active. Given that HPV is the most common sexually transmitted disease, waiting longer only increases the odds that the protection will be provided too late. The vaccine has been shown to be both safe and highly effective, and continuing experience - as of March, about 56 million doses had been distributed in the United States - reinforces that.

Yet proportional uptake in the United States has been poor. In 2010, a national survey found that only $49 \%$ of girls aged 13 to 17 had received at least one dose of vaccine, and only $32 \%$ had received all three doses. By comparison, Rwanda has achieved more than $80 \%$ vaccination coverage and several Canadian provinces have reached $85 \%$.

It is possible that unvaccinated girls in the United States are already benefiting from the compliance of the parents who have stepped up to have their children immunized. When the CDC scientists explored whether the decrease in HPV prevalence among 14-19-year-old girls might be due to herd immunity, they found that vaccinated sexually active girls showed a striking $88 \%$ decrease in prevalence of the relevant HPV strains, compared with the pre-vaccine era. But they also found a $28 \%$ decrease in prevalence among unvaccinated girls. The finding was not statistically significant, and was difficult to interpret owing to differences in the reported sexual behaviour of the two groups - for instance, the unvaccinated girls reported fewer sexual partners. Nevertheless, herd immunity is a possible explanation, and other studies have indicated that it is at play.

It is worth noting that cervical cancer, almost all of which is caused by HPV, disproportionately affects black and Hispanic women in the United States, possibly because they have reduced access to screening. And among women who do contract cervical cancer, black women have proportionally the highest death rate.

But neither white people nor parents of boys of any race or ethnicity should be complacent when considering whether to vaccinate. The
"HPV is an equalopportunity infectious agent." vaccine is not only about preventing cervical cancer, nor even only about preventing anal cancer in males who have sex with males. Consider that the proportion of US throat cancers associated with HPV has exploded in recent decades among white men and women. (Similar increases have occurred in Canada and some European countries.) As the actor Michael Douglas was frank enough to acknowledge in an interview published last month, throat cancer of the type that he was treated for in 2010 is caused by HPV contracted through oral sex. (Douglas's representatives later denied that he had intended the statement to refer to his own particular case.)

The take-home message is that HPV is an equal-opportunity infectious agent. As the CDC noted when it announced the findings last month, cervical cancer is simply the most common among about 19,000 cases of cancer caused by HPV in US women each year, and throat cancer is the most frequent among 8,000 cases of such cancers in men. The costs are sobering: the CDC calculates, for instance, that 50,000 girls alive today who will get cervical cancer during their lifetimes would not have done so had the country quickly reached $80 \%$ vaccination rates.

Squeamishness among parents being asked to vaccinate 11-yearolds against a sexually transmitted disease is understandable. But in the face of such a clearly effective means of protecting our young people, ducking the issue, hoping for the best or relying on the responsible actions of others is not.

\section{Light show}

\section{Lasers will one day improve data transfer from spacecraft, but hurdles must still be overcome.}

$\mathrm{T}$ he first images from another planet trickled to Earth as slowly as an intravenous drip. On 15 July 1965, as the spacecraft Mariner 4 swept past Mars, a small television camera began to roll. It took 22 images of the Martian surface, each 200 pixels square. Mariner 4 swivelled its high-gain, 1-metre-wide parabolic radio antenna towards Earth. It began transmitting the images in the $S$ band, a high-frequency part of the radio spectrum, at a rate of 33.33 bits per second - about a million times slower than broadband Internet connections today.

Mission scientists at NASA's Jet Propulsion Laboratory in Pasadena, California, were too impatient to wait for the complete first image, and for computers to process it. So as the data arrived, the scientists pinned ticker-tape strips of the greyscale pixel readouts to a wall - each strip a line from the image - and coloured in the rugged scene by hand with pastel crayons. It would take four days for all of the images to be returned to Earth.

Interplanetary communications have since improved considerably. The $\mathrm{S}$ band gave way to the $\mathrm{X}$ band, then to $\mathrm{K}$ and $\mathrm{Ka}$ - each at a higher frequency than its predecessor, giving engineers more cycles per second to pack in information. When the Mars Reconnaissance Orbiter (MRO) went into orbit around the red planet in 2006, it was armed with antennas that could transmit information at a rate of six megabits per second. That is a jump in Mars transmission rates of more than five

orders of magnitude in the 40 years between Mariner 4 and the MRO, and a doubling of the data-transfer rate every 2 years or so. Moore's law of computing power seems to have guided planetary data transmissions just as it has the exponential rise in transistor density on a chip.

Yet scientists, ever greedy for more data, are still constricted. The $\mathrm{MRO}$, for instance, is limited in how often it can use some of its dataintensive instruments, such as the high-resolution camera. Such bottlenecks are motivating a push for optical communications (see page 266). In the coming months, both NASA and the European Space Agency will launch spacecraft with communications modules that can use lasers rather than radio transmitters. This has two benefits. First, lasers work at higher frequencies than radio does, so they can transmit more information. Second, a laser stays collimated in a narrow beam, whereas radio transmissions spread out as they travel, meaning that lower-power laser transmitters can be used to convey the same amount of information. Optical communications can also take advantage of the extraordinary investment in lasers, which have shrunk in size and grown in power.

Scientists will surely develop instruments that take advantage of the higher bandwidth. The public could benefit, too. The NASA laser demonstration, which will go to the Moon, claims to be able to return data in high definition. Imagine seeing details of the Moon broadcast live in as much detail as TV viewers spot sweat on a football player's face.

There are issues to contend with. Lasers have a hard time transmitting through clouds, and so optical communications systems may have to depend on radio relay stations orbiting Earth (or receivers

$\rightarrow$ NATURE.COM To comment online, click on Editorials at: go.nature.com/xhunqu in cloudless deserts) for the final stretch home. But it is not too difficult to envision a day when the Solar System is stocked with spacecraft all networked by pulsed light: an interplanetary Internet. 